\title{
Être médecin et immigrant au Québec : une identité professionnelle malmenée
}

Being a Physician and an Immigrant in Quebec: A Professional Identity

Mistreated

Ser médico e inmigrante en Quebec: una identidad profesional menospreciada

Marie-Jeanne Blain, Sylvie Fortin et Fernando Alvarez

\section{(2) OpenEdition}

Journals

Édition électronique

URL : https://journals.openedition.org/remi/7021

DOI : $10.4000 /$ remi.7021

ISSN : $1777-5418$

Éditeur

Université de Poitiers

Édition imprimée

Date de publication : 1 décembre 2014

Pagination : 139-162

ISBN : 979-10-90426-23-8

ISSN : 0765-0752

Référence électronique

Marie-Jeanne Blain, Sylvie Fortin et Fernando Alvarez, "Être médecin et immigrant au Québec : une identité professionnelle malmenée », Revue européenne des migrations internationales [En ligne], vol. 30 n³ et 4 | 2014, mis en ligne le 01 décembre 2017, consulté le 14 avril 2022. URL : http:// journals.openedition.org/remi/7021; DOI : https://doi.org/10.4000/remi.7021 


\title{
Être médecin et immigrant au Québec : une identité professionnelle malmenée
}

\author{
$\ldots$ Marie-Jeanne Blain 1 , Sylvie Fortin ${ }^{2}$ \\ et Fernando Alvarez ${ }^{3}$
}

"But science and skill do not make a physician; one must also be initiated into the status of physician; to be accepted, one must have learned to play the part of a physician in the drama of medicine " (Becker et al., 2007 [1961] : 4).

\section{Introduction}

Les politiques d'immigration sont conçues pour attirer les candidats " les meilleurs et les plus brillants " dans un contexte de forte compétition internationale visant ces cerveaux mobiles (CIC, 2011). À l'instar de l'Australie, des États-Unis ou de l'Europe, le Canada s'inscrit dans ce " marché mondial des compétences médicales " (Séchet et Vasilcu, 2012). Si la "fuite " des médecins soulève des enjeux éthiques dans les zones mal desservies selon I'OMS (2010), la question de leur reconnaissance professionnelle se pose aussi au Québec et au Canada (Blain et al., 2012). En effet, les stratégies destinées à attirer une migration permanente qualifiée relèvent d'une politique gouvernementale qui les incite à s'établir, mais une fois sur le territoire, les médecins sont confrontés à de multiples défis entravant leur intégration professionnelle ${ }^{4}$. Cette situation est d'autant plus critique que la province du Québec fait face à une pénurie de médecins.

1 Doctorante, Département d'anthropologie, Université de Montréal, C.P. 6128, succursale Centre-ville, Montréal (Québec) H3C 3J7, Canada ; mj.blain@umontreal.ca

2 Professeure agrégée, Département d'anthropologie, Université de Montréal, C.P. 6128, succursale Centre-ville, Montréal (Québec) H3C 3J7, Canada ; sylvie.fortin@umontreal.ca

3 Professeur titulaire, Département de pédiatrie, Université de Montréal, CHU SainteJustine, 3175, chemin de la Côte-Sainte-Catherine, Montréal (Québec) H3T 1C5, Canada ; fernando.alvarez@umontreal.ca

4 La reconnaissance du titre de médecin varie d'un pays à l'autre et dépend des politiques en vigueur, des mécanismes corporatifs et du marché de l'emploi (Dumont et Zurn, 2007 ; Shuval et Bernstein, 1997). Au Canada, elle relève du pouvoir de la province. Le Québec affiche le plus bas pourcentage de diplômés internationaux en médecine par rapport aux autres provinces : $12 \%$ pour $25 \%$ en moyenne pour l'ensemble du Canada (ICIS, 2013). Dans les pays de I'OCDE ce taux est de $18 \%$, dont la France (17\%), les ÉtatsUnis (24\%), le Royaume-Uni (33\%) (Dumont et Zurn, 2007). 
Leurs compétences, pourtant hautement valorisées initialement, sont reconnues de façon variable. Au Canada, les démarches de reconnaissance professionnelle des diplômés internationaux en médecine (DIM) ${ }^{5}$ peuvent être très contraignantes. Pour un migrant universitaire, et plus particulièrement pour les diplômés en santé comme les médecins, l'objectif de poursuivre sa carrière dans le pays hôte peut éventuellement se réaliser ${ }^{6}$, mais s'il n'appartient pas à une filière académique prestigieuse, en tant que professeur et chercheur, sa démarche relèvera du " parcours du combattant" (Chicha, 2009)7. Aux niveaux gouvernemental et institutionnel, les politiques visant à attirer des migrants ainsi que les mesures et programmes destinés à favoriser l'intégration socioprofessionnelle des personnes immigrantes ${ }^{8}$ mettent l'accent sur l'individu, ses caractéristiques personnelles et sa capacité d'adaptation (Chicha et Charest, 2008). Cette vision idéaliste de l'intégration des migrants, selon laquelle leur capital humain et leurs choix détermineraient leurs trajectoires individuelles, fait cependant abstraction des normes et des contraintes sociales (Smith et Favell, 2006).

Par-delà les enjeux d'ordre socio-politique, que signifie être médecin et immigrant au Québec au regard de leur carrière ? À la différence d'études qui s'inscrivent dans un courant psychosocial où un fort engagement professionnel constituerait un facteur déterminant dans la poursuite d'une carrière (Remennick et Shakhar, 2003), notre recherche porte sur le caractère relationnel et stratégique du processus d'intégration socioprofessionnelle et, dans cet article, sur I'identité professionnelle. Notre approche s'inspire des travaux de Sainsaulieu (1997; 1977) en sociologie des organisations et de Shuval (2000) en sociologie de la santé, tout en privilégiant une approche anthropologique, ancrée dans les expériences de vie des personnes rencontrées et les pratiques observées.

Nous verrons comment au cours de ces démarches complexes visant la reconnaissance professionnelle, l'identité professionnelle des médecins est bien souvent malmenée. Les conditions d'appartenance formelle à la profession

5 Les termes " médecin diplômé à l'étranger ", " diplômé international en médecine " (DIM) ou " médecin diplômé hors Canada et États-Unis (DHCEU) " sont considérés ici comme synonymes. Les DIM de notre enquête sont tous migrants et diplômés hors Canada et États-Unis. Ils ont été recrutés par des institutions depuis l'étranger ou ont migré sans emploi confirmé.

62435 DIM ont leur permis de pratique au Québec (CMQ, 2013). Ils seraient 3000 non reconnus selon une association de DIM (Sow, 2010).

$7 \mathrm{Au}$ Québec, à compétence égale, un migrant qui accède à un emploi a les mêmes privilèges qu'un natif. La citoyenneté ou le statut migratoire n'a pas d'effet sur le salaire perçu par les DIM une fois en poste (on notera que la médecine est l'une des professions les mieux rémunérées au Québec). Sommairement, la durée du processus pour acquérir le permis restrictif (professeurs et spécialités en demande) est de un à trois ans ; pour I'obtention du permis régulier, le DIM doit compléter sa résidence au Québec (formation postdoctorale, équivalent à l'internat en France), et le processus est de cinq à dix ans. Sauf exception (tel L'arrangement de reconnaissance mutuelle France-Québec signé en 2009), les omnipraticiens doivent choisir la procédure d'attribution du permis régulier (Blain et al., 2012).

8 Inscrits dans la politique québécoise, il s'agit de programmes pour favoriser l'intégration des personnes immigrantes, particulièrement des nouveaux arrivants; un volet de ces derniers concerne la reconnaissance professionnelle et l'intégration au marché du travail. Ces mesures sont appliquées par des institutions gouvernementales ou des organismes communautaires financés par le gouvernement (Chicha et Charest, 2008). 
médicale déterminent fortement les trajectoires d'intégration des diplômés internationaux en médecine, bien au-delà de leur vocation et de leur engagement. Le caractère dynamique et relationnel de l'identité professionnelle, sa fluidité et ses limites est mis en relief, mais surtout, dans le cas des médecins, la puissance des conditions d'appartenance, qui obligent à une flexibilité professionnelle et parfois au retrait. À partir de témoignages de migrants, nous explorons l'identité professionnelle pour soi, puis les aspects relationnels de l'identité, c'est-à-dire les conditions d'appartenance et la catégorisation. Enfin, nous soulevons les différentes stratégies mobilisées par les migrants, lesquelles varient selon les contextes. En conclusion, nous soulignons le rôle central des notions de pouvoir et de reconnaissance au cœur des processus identitaires (Sainsaulieu, 1997 ; 1977) avant de poser un regard critique sur les approches centrées sur l'individu.

\section{Contexte de la recherche}

\section{Méthodologie}

Les résultats présentés sont le fruit d'une recherche doctorale en anthropologie menée au Québec (2009-2012) ${ }^{9}$ qui relève d'une démarche phénoménologique et interactionniste. L'objectif principal est de documenter les processus complexes à l'œuvre au cours des trajectoires d'intégration socioprofessionnelles, et plus spécifiquement les stratégies et ressources mobilisées par des DIM sur le marché du travail.

Des entretiens semi-dirigés ont été menés auprès de trente-et-un médecins diplômés à l'étranger (quinze femmes et seize hommes), âgés de trente-trois à cinquante-cinq ans et parlant français. Tous sont immigrants ayant un statut régularisé comme résident permanent (de catégories diverses : sélectionnés, réunification familiale ou réfugiés), ils vivent au Québec depuis six ans en moyenne. Les lieux d'origine et les pays d'obtention du diplôme de médecine sont très variés ${ }^{10}$. Les régions d'obtention du diplôme de médecine sont ${ }^{11}$ : Europe de I'Ouest (huit), Europe de I'Est (trois), Amérique latine (neuf), Antilles (Cuba et Haïti) (trois), Maghreb (quatre) et Afrique subsaharienne (quatre). Les entretiens étaient centrés sur les récits de vie professionnelle pré et post-migratoire. Avant la migration, les deux tiers travaillaient en tant qu'omnipraticiens (vingt sur trente-et-un), parmi ces derniers, sept travaillaient dans la santé publique ou I'administration ; I'autre tiers était composé de professeurs (cinq) ou de médecins spécialistes (six). Au moment de l'enquête, quinze ont intégré la profession médicale au Québec (ou s'apprêtaient à le faire). Ceux-ci, que nous nommons les " requalifiés ", ont obtenu des postes de professeurs (cinq),

\footnotetext{
9 Recherche menée par Marie-Jeanne Blain, sous la direction de Sylvie Fortin et Fernando Alvarez. Nous remercions le Conseil de recherches en sciences humaines du Canada et la Fondation du CHU Sainte-Justine pour leur appui, ainsi que tous les répondants. Notre enquête a été approuvée par les comités d'éthique à la recherche du CHU Sainte-Justine et de I'Université de Montréal.

10 À l'instar du portrait de l'immigration au Québec, la même diversité était souhaitée dans notre étude.

11 Pour trois répondants, le pays de naissance est différent du pays d'obtention du diplôme. Ce dernier a été retenu, alors que le processus de reconnaissance au Québec ne tient compte que du lieu des études.
} 
médecins spécialistes (deux), médecins de famille (trois), étaient en résidence (quatre) ou en démarches pour l'obtention du permis de pratique comme médecin (un). L'autre moitié (seize) a changé de carrière et s'est réorientée vers des professions non médicales. À l'exception d'une personne qui s'est dirigée vers des études supérieures en sciences sociales et d'une autre sans expérience professionnelle au Québec, tous se sont "réorientés " dans le domaine de la santé, soit dans le milieu non clinique ${ }^{12}$ - nommés "réorientés niveau universitaire en santé ": postes de niveau universitaire suite à des études supérieures (six) ou aux études supérieures en santé non clinique (cinq) -, soit en santé clinique comme techniciens (trois). Au cours de leurs trajectoires d'intégration professionnelle, les deux tiers des "réorientés " ou "requalifiés " ont occupé un emploi déqualifié, les professeurs faisant majoritairement exception.

Ce corpus a été complété par des entretiens informels menés auprès de vingtdeux personnes, dont onze acteurs institutionnels (responsables de l'embauche, représentants d'organismes d'aide à I'emploi, d'associations de DIM, etc.) et onze DIM très récemment immigrés ou projetant $d^{\prime}{ }^{\prime i m m i g r e r}{ }^{13}$. Nous avons aussi réalisé des observations ponctuelles dans le milieu associatif médical, dans le milieu communautaire (au sein d'associations pour la reconnaissance des DIM ou d'organismes d'aide à l'emploi dédiés à l'intégration des nouveaux arrivants) ainsi que lors de réunions d'information du Collège des médecins s'adressant aux DIM.

\section{Des processus d'intégration socioprofessionnelle complexes}

Les difficultés d'intégration professionnelle des DIM sont bien documentées au Québec (Bardaï, 2010 ; Chicha, 2009), mais la question qui concerne la réorientation de carrière de migrants très qualifiés et leurs processus décisionnels reste encore peu explorée. Au Canada, les DIM se distinguent d'autres groupes occupationnels par la complexité et la fragmentation des démarches de reconnaissance professionnelle (Blain et al., 2012). En plus d'être touchés par le chômage, les diplômés internationaux en santé, et spécialement les médecins, ont un accès plus limité à leur profession - $45 \%$ des DIM seraient réorientés dont plus du tiers dans des postes qui ne sont ni liés à la médecine ni aux soins de santé (Boyd et Schellenberg, 2007) -, et connaissent une déqualification importante. Ils se requalifient plus lentement comparativement à $d^{\prime}$ autres professions dont les diplômes ont été obtenus à l'étranger (Galarneau et Morissette, 2004).

Aucune étude statistique n'a encore été publiée au Québec concernant les différences d'intégration professionnelle selon l'origine nationale des DIM. Cependant, des pistes se dégagent de données canadiennes indiquant que les DIM originaires de pays francophones ou anglophones occuperaient un poste de médecin en plus forte proportion (Boyd et Schellenberg, 2007) tandis que

12 Par exemple, conseiller dans le secteur pharmaceutique, en santé et sécurité, en bioéthique, professionnel de recherche, etc.

13 Compte tenu du temps de requalification requis, sont exclus de notre population principale ces migrants très récents (moins de deux ans). Les échanges informels permettaient de recueillir leurs premières impressions. 
I'admission en résidence ${ }^{14}$ semble favorisée pour les DIM diplômés de pays occidentaux (CaRMS, 2013). L'invisibilité statistique et le peu de précision des données sur les DIM au Québec ne permettent cependant pas de donner plus de détails.

En cas de non-reconnaissance professionnelle, les migrants peuvent suivre une nouvelle formation, accepter un emploi transitoire, se diriger vers l'entrepreneuriat, redéfinir leurs attentes, rentrer au pays d'origine, ou encore aller dans un pays tiers (Chicha, 2009 ; Collins, 2012 ; Shuval, 2000). En ce qui concerne les DIM, il a été fréquemment observé que pour se rapprocher de leur profession ceux-ci tentent d'occuper un poste dans le secteur de la santé ou dans la recherche. Néanmoins, le fait de travailler en santé clinique dans un poste autre que la médecine implique un retour aux études ainsi que des démarches de reconnaissance auprès de l'ordre professionnel concerné, parmi vingt-quatre professions réglementées en santé $(\mathrm{CIQ}, 2012)^{15}$. De plus, compte tenu du cloisonnement des champs professionnels, rien ne garantit au DIM que I'emploi transitoire soit un tremplin vers la médecine, même si travailler dans un poste en santé peut permettre de mettre à profit ses compétences, de se familiariser avec le système de santé québécois ou simplement d'exercer une profession apparentée à celle exercée auparavant.

\section{Identité et stratégies identitaires}

Pour Sainsaulieu (1977), la reconnaissance de l'identité s'inscrit dans un ordre établi qui s'ancre dans " l'expérience relationnelle et sociale du pouvoir". II en résulte une identité " pour soi " et une identité " attribuée " qui peuvent être en désaccord et seront négociées par les acteurs en présence (Goffman, 1975 ; Dubar, 1995 ; Sainsaulieu, 1977). L'identité n'étant jamais donnée une fois pour toutes, la notion d'identification serait plus juste ; elle met en relief son caractère construit dans lequel des enjeux de pouvoir sont sous-jacents (Gallissot, 1987). Cette conception permet de dépasser l'aspect psychologique de l'identité et de tenir compte des dimensions liées aux conditions d'appartenance et à la catégorisation. Les stratégies identitaires quant à elles se situent au confluent des dimensions micro et macro-sociales. Elles sont influencées par les situations d'interaction, mais ne dépendent pas uniquement de ces dernières - voir les stratégies de "résistance au stigmate " (Goffman, 1975) ou le "revirement du sens de l'épreuve " (Fournier et Bourassa, 2004). Cette capacité stratégique, cette possibilité de faire des essais et erreurs, est inégalement répartie et dépend des relations de pouvoir et de la position de chacun des acteurs (Sainsaulieu, 1977), de ses ressources sociales et symboliques (Berset et al., 1999 ; Fortin et Renaud, 2004).

14 La réussite de la résidence au Québec (formation postdoctorale équivalant à l'internat en France) est obligatoire pour I'obtention du permis régulier de pratique pour les DIM non admissibles au permis restrictif comme spécialiste ou professeur. Les résidents reçoivent un salaire durant leur formation. Les places en résidence sont contingentées, certaines spécialités plus que d'autres. Le taux moyen de réussite à l'admission des DIM au Québec entre 2010 et 2013 est de 30 \% (CMQ, 2013), même s'ils ont obtenu au préalable une équivalence de leur diplôme par le Collège des médecins du Québec. Le fait que plusieurs universités préfèrent laisser des postes de résidents vacants suscite de vifs débats dans le milieu pour la reconnaissance des DIM, « les facultés de médecine [...] répliquèrent qu'elles préféraient laisser les postes vacants, estimant que les médecins DHCEU n'ayant pas été jumelés ne possédaient pas les "qualités requises" " (CDPDJ, $2010: 2$ ).

15 À la différence de la France, par exemple, où un DIM peut exercer comme « praticien associé " ou infirmière (Dumont et Zurn, 2007). 
La profession procure un sentiment d'appartenance à une communauté partageant un ensemble de normes et de valeurs (Dubar, 1995 ; Shuval, 2000). Suite à un long processus de socialisation, la profession médicale, même subdivisée en plusieurs spécialités, peut procurer ce sentiment de partager une culture professionnelle commune (Becker et al., 2007 ; Good et DelVecchio, 1993). Une étude récente menée auprès de DIM luttant pour la reconnaissance de leur titre de médecin au Canada propose même l'existence d'une " diaspora professionnelle ", au sein de laquelle l'identité professionnelle de médecin rassemble des individus d'appartenances, de cultures et de langues diverses (Neiterman et Bourgeault, 2012). Selon ces auteures, I'identité professionnelle antérieure de ces médecins ainsi que les incertitudes entourant leur avenir dans le milieu médical canadien les unissent et contribuent à créer une communauté d'entraide composée de DIM de toutes origines. Dans le cadre de notre recherche, I'identité de DIM transparaît notamment pour ceux qui ont un " parcours du combattant " pour la reconnaissance. Dans leurs cas, I'appui des pairs s'avère central pour parvenir à une reconnaissance professionnelle, non seulement pour faire face à la lourdeur et à la difficulté des démarches à entreprendre, mais aussi pour mettre en œuvre des stratégies efficaces - par exemple l'accès à des informations non officielles essentielles comme les stages d'observation préalables à la résidence (Blain et al., 2012). Toutefois, dans notre recherche nous mettons en lumière une situation pour le moins paradoxale, puisque certains DIM se distancient de cette identité de groupe.

Des recherches menées auprès de médecins immigrants ont mis en évidence des liens entre les notions d'identité professionnelle et d'engagement (professional commitment) (Remennick et Shakhar, 2003 ; Shuval, 2000 ; Shuval et Bernstein, 1996). Un médecin très engagé et très lié émotionnellement à sa profession chercherait davantage à être reconnu après son arrivée au pays hôte. Remennick et Shakhar (2003) évoquent une identité professionnelle peu malléable, à I'exemple de DIM reconvertis en physiothérapeutes, mais se définissant toujours comme médecins malgré leurs nouveaux statut et rôle. Nos résultats soulignent au contraire que l'identité professionnelle des médecins s'avère flexible dans certaines situations, en particulier s'ils puisent dans des ancrages identitaires antérieurs, c'est-à-dire dans les cas où ils se considéraient polyvalents et avaient occupé au préalable des fonctions hors du domaine de la médecine clinique ou, dans d'autres cas, s'ils ont le sentiment de poursuivre leur spécialité médicale à travers une nouvelle carrière au Québec. Au reste, comme nous le verrons, nos résultats indiquent que cette flexibilité identitaire trouve ses limites lorsque le DIM se réoriente au Québec dans une profession technique. Par ailleurs, la notion d'engagement telle qu'avancée par Remennick et Shakhar (2003) ne peut à elle seule expliquer des phénomènes d'auto-retrait de la profession médicale qui ont été documentés (Belkhodja et al., 2009 ; Chicha et Charest, 2008 ; Hachimi Alaoui, 2007). En effet, le fort corporatisme décourage plusieurs migrants à entamer des démarches de reconnaissance de leurs acquis professionnels, en dépit de leur sentiment de vocation professionnelle vis-à-vis de la médecine. En ce sens, la perspective que nous retenons est celle de Judith Shuval (2000) : la notion d'engagement peut éclairer certaines stratégies d'intégration professionnelle, mais ce phénomène complexe doit être analysé à la lumière des conditions structurelles d'accès à la profession médicale. 


\section{L'identité professionnelle pour soi}

"Être médecin, ce n'est pas juste avoir un diplôme, c'est viscéral, c'est au fond de nous " (Véronique16, France, réorientée niveau universitaire en santé).

Ces médecins ont choisi leur profession pour différentes raisons : I'influence d'un moment clé vécu durant l'adolescence, un proche œuvrant dans le milieu médical, le besoin de se sentir utile, l'attrait pour les aspects scientifiques ou humains, etc. La formation est exigeante et longue et, même si certains pressentaient les nombreux défis associés à une intégration au système médical québécois, peu ont laissé de plein gré leurs carrières, quels que soient leurs motifs d'immigration. En effet, au cours du processus d'immigration, tout médecin doit signer une lettre déclarant qu'il est informé des difficultés d'accès à la profession médicale au Québec ${ }^{17}$. Bien qu'informés de cette situation, au moment de migrer, certains pensent se faire une place malgré tout, $d^{\prime}$ autres sont prêts à mettre leur profession " en berne " tandis que d'autres désirent tenter leur chance avec la possibilité de repartir si l'expérience ne leur convient pas.

Quant à la vision de leurs trajectoires, dans la catégorie des "requalifiés ", il y a ceux pour qui cela représente une mobilité professionnelle, "les recrutés ". Ce sont dans notre population d'enquête les professeurs (et un spécialiste), tous recrutés depuis la France qui ont immigré munis d'une lettre d'embauche provenant d'un établissement québécois et qui ont bénéficié d'une reconnaissance relativement rapide du Collège des médecins (un à deux ans environ). Pour les autres "requalifiés ", que nous nommons "les batailleurs ", cela s'est fait au prix de démarches intenses, semées d'embûches. Le processus est éprouvant tant du point de vue humain que financier et s'étale sur plusieurs années (entre trois et dix ans) : via l'obtention du permis restrictif comme spécialiste ou suite à la résidence. Pour ces derniers, il peut y avoir des "compromis" professionnels (un spécialiste opte pour la médecine familiale ou un professeur surspécialisé devient simple spécialiste).

Dans le groupe des "réorientés ", deux tendances se dégagent. Certains vont insister sur la continuité de leur carrière dans leur nouvelle profession. Cette perception de continuité se fera soit via une valeur ajoutée accordée à la spécialité ou au domaine d'expertise exercé avant la migration (la santé publique par exemple), par un intérêt particulier que l'on avait (comme la bioéthique), ou par la poursuite d'un projet qui commençait à se former avant leur départ (comme la médecine alternative). Pour d'autres, le changement de carrière représente un choix de compromis, par manque d'option, par " résignation " selon Chicha (2009). II s'agit d'adopter une carrière dans le domaine de la santé qui requiert un bref investissement de temps et un emploi convenable à court terme (les techniques en santé).

16 Les noms sont des pseudonymes.

17 Déclaration d'un médecin diplômé hors du Canada et des États-Unis (www.immigration-quebec.gouv.qc.ca/publications/fr/dcs/D04.pdf). 


\section{L'identité fluide}

"Ce n'est pas évident que si tu changes de pays, ton statut professionnel change aussi. Dans ta tête, tu cherches à garder toujours le même statut: "Si je suis médecin ici, pourquoi pas là-bas ?" " (Leandro, omnipraticien en Colombie, réorienté technicien en santé).

Le processus identitaire est dynamique et l'individu peut réinterpréter à tout moment sa trajectoire et modifier conséquemment ses stratégies professionnelles. La vision de soi peut changer au fil du temps et les médecins rencontrés nous en donnent maints exemples. La médecine clinique peut devenir secondaire tout en conservant ses acquis comme professionnel en santé (sans la clinique) ou le DIM peut s'identifier à une nouvelle profession. Türegün (2011) parle de reconstruction professionnelle et Shuval (2000) de nouvelle identité professionnelle. Dans notre recherche, nous avons identifié deux cas de figure en ce qui concerne la réorientation, I'une positive et l'autre ambiguë, voire même négative.

Cette reconstruction apparaît positive pour ceux dont une professionnalité est reconnue et qui puisent dans des ancrages identitaires antérieurs, c'est-àdire ceux qui avaient exercé des fonctions hors de la santé clinique avant la migration ou qui éprouvent un sentiment de continuité de leur spécialité. C'est le cas des DIM occupant des professions de niveau universitaire en santé publique, bioéthique, santé et sécurité au travail, etc. "Je me sens valorisée comme professionnelle ", dira Carmen (Venezuela). Ils ont davantage le sentiment de poursuivre leur carrière, que ce soit en exerçant différemment leur spécialisation ou en travaillant dans un domaine qui leur est familier, auquel ils s'identifiaient déjà avant la migration. Ces répondants ont entrepris ou complété des études supérieures qui ont pu donner une valeur ajoutée à leur formation médicale de base. Plusieurs ont mentionné avoir été embauchés précisément parce qu'ils sont (étaient) médecins. Un sentiment de continuité et de reconnaissance de leur expertise est ressenti. Néanmoins même pour ces répondants, la précarité d'emploi ou le sentiment de ne pas être rémunéré à leur juste valeur peut mettre un bémol à ce relatif " confort identitaire ".

La reconversion dans des postes techniques nous permet de cerner les limites de la flexibilité identitaire. Le sentiment de malaise est particulièrement exacerbé pour les DIM ayant fait un choix de compromis en technique de la santé et dont le nouveau rôle ne donne pas accès à une autonomie décisionnelle et à une reconnaissance de leur professionnalité. L'acquisition d'une nouvelle identité professionnelle en technique de la santé peut impliquer une " régression " difficile à accepter (Collins, 2012). En effet, l'individu puise dans ses ressources, mais est contraint par des limites, notamment structurelles ; le choix professionnel "par résignation " en témoigne. L'identité de médecin se cristallise lorsque cette nouvelle identité professionnelle leur est étrangère ou lorsque leurs interactions quotidiennes rappellent ce qui a été perdu (Vapor et $\mathrm{Xu}, 2011)$. Les migrants rencontrés ne semblent pas avoir intégré de façon positive ce nouveau statut, peut-être parce qu'il s'agit d'un choix de compromis afin de poursuivre a minima dans le domaine de la santé ou encore, du fait que la profession n'est pas de niveau universitaire. Sofia, spécialiste en Ukraine et étudiante en techniques infirmières depuis un an et demi, en témoigne : " J'ai 
compris que ce n'était pas un bon choix. [...] Ce n'est pas mon niveau. Je peux faire plus. J'ai des connaissances plus larges et je ne vais pas me respecter dans le niveau d'infirmière ". Elle envisage d'interrompre ses études pour entamer le processus de reconnaissance comme médecin, quatre ans après son arrivée au Québec.

Se retrouver dans une filière technique, non valorisée, dans une structure décisionnelle où le médecin agit encore souvent comme figure centrale-malgré la professionnalisation des autres carrières en santé (Dussault, 1985) - pourrait exacerber l'identité vocationnelle de médecin et le malaise lié au sentiment de ne pas être à sa place. L'identité peu malléable des médecins reconvertis comme physiothérapeutes en Israël trouve ici écho (Remennick et Shakhar, 2003). Leur difficulté à se réorienter, attribuée par les auteures à un fort engagement vis-à-vis de la médecine, s'explique également, selon nous, par leur professionnalité non reconnue. La capacité limitée de poser un jugement clinique pourra s'avérer frustrante pour des médecins aujourd'hui techniciens en santé. Par exemple, Véronique qui avait envisagé d'être infirmière s'est ravisée : "Si je me trouve face à un médecin qui prescrit quelque chose et que moi, en tant que médecin, quand même, je ne suis pas tout à fait d'accord, je risque d'avoir des problèmes pour vivre ça, et donc je n'ai pas voulu choisir cette voie-là " (France, réorientée niveau universitaire en santé). À l'instar des résultats de Vapor et Xu (2011 : 218), cette transition professionnelle de médecin à infirmière, de " "thinker" (prescribing orders) in medicine to a "doer" ", suscite des émotions négatives : une baisse d'estime de soi, de la gêne, une perte d'identité et une difficulté à renoncer au rôle de médecin. Ces DIM techniciens sont constamment rappelés " à la personne qu'on aurait aimé être, la personne qu'on était " (Sayed, Algérie, médecin de famille au Québec). Citons Sofia, réorientée comme technicienne en santé, qui rapporte l'expérience d'un ami autrefois chirurgien :

" II m'a dit: "Quand je travaille comme préposé dans le département de chirurgie [il était chirurgien], le cœur déchire dans ce moment, parce qu'il fait la même chose que j'ai faite, mais je ne peux pas regarder dans le dossier, je ne peux rien faire... c'est dur". Et il retourne à la maison chaque fois déçu... et chaque fois il recommence... " (Sofia, spécia-

liste en Ukraine, réorientée technicienne en santé).

\section{Stratégies d'ajustement de la vision de soi et relativisation}

"Et je pense que... je suis toujours médecin, en tout cas, profondément " (Isabelle, spécialiste en France, réorientée niveau universitaire).

Les stratégies déployées par les DIM " réorientés " nous semblent constituer des " stratégies de protection identitaire ", c'est-à-dire des façons de donner sens à son parcours, de le réinterpréter. Un sens est donc donné à l'occupation de ces emplois, et l'identité est ajustée en conséquence. En cas de non-reconnaissance, deux stratégies sont mobilisées. Le DIM pourra se considérer toujours médecin, malgré tout, ou bien il pourra relativiser son nouveau rôle professionnel en mettant l'accent sur la dignité de tout travail ou la priorité accordée à la famille.

Une stratégie récurrente consiste à maintenir son identité professionnelle, malgré sa non-reconnaissance au Québec. Carmen, réorientée, a réfléchi à cette question : "Parfois je m'entends dire "je suis médecin au Venezuela". Mais je ne suis pas médecin au Venezuela, je suis médecin simplement, tu comprends ?" 
Ils insistent sur le fait qu'ils sont toujours médecins, mais ailleurs. II y a un poste en attente au pays d'origine, ou bien ils y maintiennent leur permis de pratique valide s'ils décident une migration de retour. "Je sais que si je retourne en France, je vais exercer - si je veux " (Isabelle). Certains peuvent également se dire qu'il est toujours possible de travailler à l'international et " on n'aura rien perdu " (Leandro). Pour les DIM réorientés, cette capacité de se dire et de se voir toujours médecin, par-delà les frontières, permet de maintenir une vision de soi, malgré I'identité attribuée localement.

Dans les cas où l'emploi occupé représente une déqualification importante, I'accent est mis sur la valeur du travail, qui permet de faire vivre dignement sa famille, de vivre de façon autonome (voir Vapor et $X u, 2011$ ). Sayed sans emploi durant une certaine période, vivant de prestations de dernier recours du gouvernement, nous dira qu'un jour il en a " eu marre » et qu'il a accepté n'importe quoi :

" J'ai dit: "C'est quoi le travail ?". On m'a dit: "Du travail physique. Êtes-vous capable ?". "Oui, je suis capable". [...] Bien je suis allé prendre des cartons ! Puis c'est tout. Et je ne suis pas mort. Et je n'ai pas honte de le dire, au contraire, j'en suis fier "

(Sayed, Algérie, aujourd'hui médecin de famille).

D'autres relativisent leur position, concluant qu'ils ont fait un travail comme tout immigrant "typique ", accédant à un emploi déqualifié en début de parcours. Ainsi, " la carrière de l'immigrant " (Hachimi Alaoui, 2007) permet de relativiser sa position professionnelle. "Je le prends déjà beaucoup moins personnel ", confiera Isabelle (France, réorientée) ; " je n'étais pas une exception ", évoquera Françoise (France, agente de recherche avant d'être professeure). On peut aussi comparer sa situation à ce qu'elle aurait été ailleurs, soit parce que l'on connaît des amis médecins ayant migré dans un pays tiers et "qui n'ont même pas réussi à trouver un poste comme professionnel "(Carmen), soit en se remémorant les difficultés ou le "stress " vécus au pays d'origine.

Une autre façon encore de relativiser sa situation professionnelle est de mettre en avant d'autres aspects positifs de sa vie, particulièrement la vie familiale. Ainsi, tout peut bien aller, sauf la vie professionnelle. La migration peut être au centre d'un projet familial plus large et alors, les aléas professionnels sont minorés par le bien-être de la famille (Blain, 2006 ; Vapor et Xu, 2011).

\section{Conditions d'appartenance et catégorisation}

\section{Contraintes d'appartenance et " auto-retrait »}

" Il faut le vouloir, mais il faut aussi en avoir les moyens " (Angèle, Burkina Faso, réorientée niveau universitaire en santé).

Les contraintes de la reconnaissance professionnelle sont nombreuses. Même si I'on souhaite poursuivre sa carrière, encore faut-il traverser un processus exigeant et onéreux, dont l'issue est plus qu'incertaine. Ces professionnels de la santé pourront alors opter pour un retrait du champ de la médecine, une migration de retour ou dans un pays tiers (Collins, 2012). 
Dans le cadre de notre recherche, un important phénomène d' " auto-retrait " face aux démarches très contraignantes a été constaté, soit dès le départ, soit suite à un blocage à l'une des étapes du processus de reconnaissance. C'est le cas de plus de la moitié des médecins réorientés. Par exemple, Isabelle (spécialiste en France) n'a jamais entamé de démarches pour être médecin au Québec: " je ne veux pas repasser des examens et en plus refaire [des] années d'études ". Pour Didace (République démocratique du Congo) et Rosalie (Burkina Faso), c'était " une décision qui était déjà prise ", la lettre signée à l'ambassade au cours du processus d'immigration - la déclaration du médecin DHCEU - quant à la très grande difficulté d'exercer la médecine au Québec les a immédiatement découragés. Pour Nafissa (Algérie, sans emploi au Québec), le processus est trop cher et trop long : "Même si on a la volonté, on n'a pas les moyens. Donc, j'ai opté pour femme au foyer. Je ne suis pas satisfaite, sincèrement, je me sens comme... j'ai perdu tout ". D'autres ont amorcé le processus, mais ont abandonné l'idée suite à un blocage, dont Carmen (Venezuela) qui réalise ne pas pouvoir se dédier simultanément à son emploi actuel et au processus d'accréditation, ce qui impliquerait d'étudier à temps plein (et sans ressource). Elle décide de donner la priorité à sa famille et à la stabilité financière.

D'un autre côté, dans les cas où l'identité vocationnelle est forte et qu'aucune voie de reconnaissance ne se présente, l'option du retour au pays d'origine, dans un pays tiers ou dans une autre province canadienne est fréquemment évoquée. La quasi-totalité des répondants I'a spontanément exprimée, soit pour témoigner de leurs réflexions, soit pour relater l'expérience de collègues DIM. Les requalifiés exerçant la médecine au Québec aujourd'hui se souviennent avoir examiné la possibilité d'un retour au pays lorsque le processus semblait sans issue. Eduardo (Argentine) y a pensé quand tout allait mal : "j'étais complètement déçu [...] je voulais rentrer ". Presque tous les professeurs étaient prêts à quitter s'ils n'avaient pu pratiquer. Et s'il n'avait pas débuté sa résidence dans sa spécialité, Manuel (Colombie) aurait migré avec sa conjointe dans un pays tiers. Pour d'autres, il s'agit de se donner " une sorte de délai raisonnable " (Mehdi, Algérie, réorienté niveau universitaire en santé), de conserver l'option ouverte de quitter le Québec s'ils ne trouvent pas l'emploi idéal et stable.

Or, c'est lorsque trois éléments spécifiques sont réunis qu'un malaise apparaît : une identité vocationnelle forte, la non-reconnaissance des acquis professionnels et l'impossibilité de mettre en œuvre des " voies de contournement " ou " de sortie ". En effet, pour les DIM non reconnus qui considèrent leur profession comme centrale et qui sont en quelque sorte "bloqués ", la situation est particulièrement insatisfaisante. C'est le cas de plusieurs migrants originaires de pays où la situation économique ou politique est instable, telle Nafissa (Algérie, sans emploi au Québec) qui sent avoir tout perdu ou Didace (République démocratique du Congo, réorienté technicien en santé) qui trouve " très dur et très douloureux " de ne pas avoir la possibilité de " recycler sa formation honorablement ". Nos analyses vont dans le sens de la recherche de Belkhodja et ses collègues (2009) portant sur l'intégration professionnelle de diplômés internationaux en santé. Ne pas être en mesure d'être reconnu comme médecin et se sentir " coincé " dans le pays d'accueil peut entraîner un sentiment de double échec: "Comme ils ont tout laissé dans leur pays d'origine pour venir au Canada, il n'y a pas de retour possible. Ils se sentent coincés. Ils ne peuvent pas retourner dans leur pays [d'origine], recommencer à zéro et 
admettre la faillite, mais ils n'arrivent pas à s'intégrer professionnellement ici [au Canada]. Ils ressentent l'échec des deux côtés " (Belkhodja et al., 2009 : 38).

La médecine considérée ou non comme une vocation ne coïncide pas d'emblée avec les démarches de reconnaissance entreprises une fois immigré au Québec. Le contexte de la reconnaissance joue beaucoup plus fortement que l'identité pour soi. En effet, nous avons observé un phénomène de retrait récurrent, qui se manifeste par l'auto-exclusion des démarches de reconnaissance ou par une émigration du Québec. II est clair que les DIM luttant pour être reconnus ont leur profession à cœur, d'autant plus que devenir médecin implique de nombreuses années d'investissement à plusieurs niveaux (personnel, financier, etc.). Toutefois, " ne pas entrer dans la lutte " ou ne plus se dire médecin ne signifie pas nécessairement une absence de vocation. En outre, la réorientation professionnelle entraîne clairement des stratégies identitaires, telles que la relativisation, la valorisation du nouveau poste comme professionnel ou l'accent porté sur la valeur du travail. Les cas où aucune stratégie de protection identitaire n'est possible peuvent entraîner un deuil professionnel particulièrement difficile à vivre. Le DIM choisira alors de demeurer dans le pays hôte pour des raisons autres que celles liées à sa carrière, avec un choix professionnel impliquant un certain sacrifice, assumé avec plus ou moins de bonheur.

\section{L’identité attribuée}

Explorer l'identité est indissociable de la reconnaissance et de l'attribution identitaire. Sauf exception pour la carrière universitaire, les DIM ont quasi systématiquement reçu des jugements a priori plutôt négatifs quant à leurs possibilités de pratiquer la médecine. S'agit-il de remarques implicites visant les compétences des personnes immigrantes ou de discours qui découlent de situations complexes où de nombreux acteurs institutionnels interviennent, souvent en vase clos (Blain et al., 2012) ? Nous pouvons difficilement trancher. II est toutefois frappant de constater que selon " la porte où l'on frappe " - d'où l'importance d'un réseau de contacts ou d'une bonne stratégie initiale -, les conseils vont orienter les DIM soit vers des filières très déqualifiantes (niveau non spécialisé ou technique) soit vers la reconnaissance d'une certaine professionnalité (niveau universitaire).

\section{L'exception : la carrière universitaire}

"Moi je suis en position de force, on peut dire. En tout cas, je suis un des rares spécialistes dans mon truc, personne ne va venir m'embêter " (Pierre, professeur, démarches de six mois depuis la France pour la reconnaissance).

Pour certains médecins, spécialement les professeurs-chercheurs, venir au Québec représente une mobilité socioprofessionnelle ascendante ou une amélioration de leur " qualité de vie ", en particulier quant à la charge de travail ou aux possibilités de mener leurs recherches. Ces professeurs ont bénéficié de conditions de recrutement particulières. II ne s'agit pas seulement de conditions d'accès à la pratique - le processus d'obtention du permis restrictif comme professeur étant beaucoup plus simple et rapide-, mais aussi de reconnaissance et de démarches concrètes menées par les institutions pour les attirer et les 
retenir. Ces médecins, tous recrutés en France, hommes comme femmes ${ }^{18}$, sont des spécialistes reconnus dans leurs disciplines. Ils bénéficient de ressources symboliques et relationnelles (Berset et al., 1999 ; Fortin et Renaud, 2004) tels le prestige international, les contacts établis à travers des congrès internationaux, mais aussi le fait d'être insérés dans des filières académiques valorisées.

\section{" Fais le deuil de la médecine ", "Sois flexible "}

" Dans tous les endroits que j'étais, ils m'ont dit à l'ambassade [...] : "Voilà, il faut signer le décès de votre profession". Et je l'ai signé ! Et quand je rentre ici [au Québec], je n'avais pas de travail. Bien, ils m'ont dit [au gouvernement] : "il faut être flexible"... Donc, c'est quoi être flexible? Chauffer un taxi ? Non, je trouve ça... un peu dénigrant pour... C'est quoi être flexible?" (Eduardo, Argentine, spécialiste au Québec suite à un parcours du combattant de six ans).

Les discours officiels du gouvernement et des organisations relayant ses positions sont peu encourageants à l'égard des diplômés internationaux en médecine. C'était d'autant plus fréquent avant 2002, lorsque la médecine était une profession exclue de la catégorie des immigrants sélectionnés ${ }^{19}$, mais aujourd'hui, les messages de " deuil de la médecine " et de "flexibilité professionnelle " demeurent toutefois fort présents. En effet, à moins d'être recrutés par une institution hospitalière depuis l'étranger, les médecins candidats à l'immigration sont informés par l'intermédiaire de l'ambassade, puis par les représentations du ministère de I'Immigration au Québec qu'il sera très difficile d'exercer leur métier et qu'aucune garantie ne peut leur être donnée concernant l'obtention d'un permis de pratique ou d'un accès à la résidence en médecine pour les DIM devant suivre cette voie pour la reconnaissance professionnelle. Près des deux tiers des répondants, exerçant aujourd'hui comme médecin ou non, tant avant 2002 qu'après, ont reçu explicitement ce type de message $\mathrm{e}^{20}$. Certains représentants vont relayer le message de façon un peu moins équivoque, comme en témoigne Eduardo. Ces messages officiels qui informent les médecins des difficultés qui les attendent et qui les encouragent à une certaine flexibilité professionnelle peuvent en réalité découler d'une réalité structurelle particulière (qui résulte d'un corporatisme fort). Ils peuvent témoigner d'un sentiment d'impuissance de la part des conseillers et viser à aider le nouvel arrivant à ne pas se faire d'illusions. Ils peuvent en revanche également découler d'une idéologie implicite qui considère que le médecin diplômé à l'étranger ne s'adaptera pas aisément au contexte québécois, qu'il n'est pas " à niveau ", voire même qu'il est un " médecin de seconde zone " ${ }^{21}$.

18 La situation des femmes serait à investiguer davantage, les parcours de ces professeures ont été plus longs et précaires.

19 Seuls les professeurs pouvaient être sélectionnés avant 2002. II était toutefois possible de migrer via d'autres catégories d'immigration (par exemple, famille, réfugié, conjoint du requérant principal) et de faire les démarches $d^{\prime}$ accréditation une fois établi.

20 Les migrants entrés au Canada avec un visa d'immigration temporaire comme professeur ou étudiant ont été moins exposés à ce type de message, contrairement à ceux qui ont dès le départ fait les démarches comme résident permanent.

21 " Des médecins à deux niveaux, pas plus qu'une médecine à deux vitesses ! " dira en 2005 le président du Collège des médecins (Lamontagne, 2005 : 5). 
Par exemple, lors d'une réunion d'information sur les professions réglementées, un représentant du ministère de I'Immigration a déclaré à un médecin spécialiste : "que la situation s'améliorait [...] et qu'on avait tout de même le droit d'être préposé aux bénéficiaires " (Isabelle). Plus généralement, pour tous les chercheurs d'emploi nouveaux arrivants, la position officielle du ministère de I'Immigration est d'inciter à la flexibilité professionnelle " en acceptant un emploi autre que celui visé ou comportant des responsabilités moindres, le temps d'acquérir une expérience québécoise ou de suivre une formation " (MICC, 2012 : 1). Ces messages de flexibilité et de "deuil professionnel " peuvent aussi être véhiculés par des conseillers en emploi. Lorsque Sayed affirme être médecin à son conseiller, ce dernier lui répond "oublie ça !". On déclare à Pérard et Nafissa : "vous êtes médecins, on ne peut rien faire pour vous ", ou encore à Isabelle, "il faut que vous fassiez le deuil de votre métier, vous ne serez plus jamais médecin".

Ce type de message qui est adressé par des organismes officiels n'est pas sans effet sur la réalisation du projet professionnel. Certains des médecins concernés jugent cependant que le parcours sera difficile, mais pas impossible. Pour d'autres, tout espoir de travailler un jour en tant que médecin est perdu et aucune démarche n'est même entamée: " J'étais prévenue que l'on ne voulait pas de moi " (Véronique), "la porte est fermée avant même d'entrer [...] moi, ça m'a beaucoup affectée "(Carmen). Sayed nuance le message reçu lors de son entretien d'immigration à I'ambassade canadienne: on ne lui a pas dit que c'était impossible, mais "presque impossible ". II ajoute "quand je suis arrivé ici [au Québec], je ne rêvais pas, non. J'ai été m'inscrire à des cours d'informatique " (Sayed, Algérie, médecin de famille au Québec).

Bien que ce type de propos ne reflète pas la position de tous les conseillers, il semble néanmoins s'inscrire dans un contexte idéologique institutionnalisé (Chicha et Charest, 2008). Pourtant, un emploi transitoire peut s'avérer être autant un tremplin qu'un cul-de-sac. Alors que le temps constitue un facteur central pour la réalisation du projet de requalification des médecins, les conseils obtenus en début du parcours jouent un rôle essentiel (Chicha, 2009). L'accumulation de démarches inappropriées et menant à l'échec peut décourager sinon bloquer le chercheur d'emploi (Blain, 2006).

\section{Reconnaissance différenciée selon les contextes}

II est manifeste que la reconnaissance du bagage professionnel des DIM varie selon les contextes ou selon la personne à laquelle ils s'adressent. Un médecin par exemple sera reconnu dans son pays d'origine par ses collègues canadiens en santé internationale, mais pas sur le sol québécois. Au Québec, selon l'endroit où le DIM se dirige, les conseillers ou les responsables des ressources humaines accorderont plus ou moins de valeur à ses acquis professionnels, lui conseillant de suivre une voie déqualifiante, ou au contraire de se diriger vers des filières où son diplôme est davantage valorisé.

Vers quelle profession s'orienter si I'on est médecin diplômé à l'étranger et que l'on ne peut pas exercer? En plus des conseils prônant le deuil et la flexibilité professionnelle, une orientation couramment proposée est celle d'un emploi du même domaine, en santé. Dans ce contexte, deux éléments sont à considérer. 
D'une part, étant donné que les professions en santé clinique sont réglementées, se réorienter dans ce champ implique un retour aux études et des démarches de reconnaissance vis-à-vis de l'ordre professionnel concerné. En outre, exercer d'autres emplois en santé implique quasi nécessairement une profession " subordonnée " à la médecine, sauf si le DIM envisage des études de maîtrise ou de doctorat et l'abandon de la pratique clinique. En effet, " historiquement, cette structure a pris la forme d'une hiérarchie d'occupations dans laquelle la médecine occupe une position structurellement dominante " (Dussault, 1985 : 588), tant symboliquement que du point de vue de la rémunération.

II ressort des entretiens que des conseillers - agent d'immigration, conseiller en emploi ou responsable des ressources humaines - orientent de façon récurrente les médecins étrangers vers des postes tels que préposés aux bénéficiaires ou infirmières. II peut s'agir de conseils avertis, permettant d'accéder à une situation professionnelle moins incertaine que la médecine. Pour les DIM consultant des organismes chargés de l'emploi, de telles orientations vers des postes techniques peuvent aussi s'expliquer par la structure des programmes gouvernementaux qui priorise l'intégration en emploi rapide et le recyclage professionnel de courte durée (Chicha et Charest, 2008).

Du point de vue des intéressés, un sentiment de non-reconnaissance domine. Ce dernier résulte de l'inadéquation entre ce qu'on leur propose et leur identité de médecin et ce, d'autant plus que le poste de préposé aux bénéficiaires "l'échelon le plus bas "22 -, semble parfois la seule option envisageable dans le secteur de la santé. De nombreux DIM ont connu ce type de reconnaissance parcellaire :

"Et là, un représentant d'un établissement [de santé] m'avait dit : "Ah, préposée aux bénéficiaires, c'est tout ce que je peux vous proposer. Votre diplôme ne vaut rien"... Oui.

On est humiliés en fait " (Véronique, France, réorientée niveau universitaire en santé).

À la différence des situations que nous venons de décrire, des responsables de programmes d'études supérieures à l'université (deuxième cycle) proposent d'autres options dans le but de mettre à profit les compétences des migrants. Certains programmes d'études supérieures reconnaissent le niveau de scolarité du diplôme de médecine, tels que bioéthique, santé publique, santé et sécurité au travail, etc. En contraste, I'accès au premier cycle universitaire s'avère généralement plus complexe en raison du contingentement des programmes en sciences de la santé (tels que sciences infirmières, nutrition ou ergothérapie). Dans notre enquête, les DIM réorientés qui occupent aujourd'hui des postes de niveau universitaire ont tous complété des études supérieures au Québec qui leur ont permis de mettre à profit leurs compétences et d'accéder à des postes mieux rémunérés.

Pourquoi une telle différence dans les orientations suggérées par des conseillers à ces DIM ? Entre un poste de préposé aux bénéficiaires et celui de conseiller en santé publique dans une agence reconnue, il existe un écart important en termes de salaire, de prestige, de responsabilité et de reconnaissance sociale. La source des conseils reçus, le type de ressources consultées et par ricochet,

22 Expression de Claudia (Colombie, en résidence au Québec). 
le réseau d'informations des DIM, ont une forte influence sur les trajectoires professionnelles ; à ce titre, les ressources sociales et symboliques sont déterminantes.

\section{Stratégies de présentation de soi}

\section{Identité professionnelle contextuelle : dire ou ne pas dire que l'on est médecin}

« Non jamais ! Jamais ! Jamais ! Même maintenant, personne ne sait à I'hôpital que je suis médecin " (Leandro, omnipraticien en Colombie, réorienté technicien en santé).

À qui dira-t-on que I'on est médecin lorsqu'on occupe un emploi qui s'en éloigne ? "Si je suis préposée aux bénéficiaires, comment mes collègues pourraient réagir s'ils l'apprenaient? ". "Et devrais-je le mentionner pour l'obtention d'un emploi d'agent de recherche? ". Dans le cas des DIM concernés, on observe différentes façons de se présenter face à des situations où la question de dire ou ne pas dire que l'on est médecin se pose.

Pour les nouveaux arrivants, ne pas faire état de la profession antérieure afin de ne pas être écarté d'un poste pour lequel ils seraient jugés surqualifiés est une stratégie courante (Blain, 2006 ; Chicha, 2009). Belkhodja et ses collègues (2009) mentionnent que certains diplômés internationaux en santé " dévalorisent leur curriculum vitae " afin d'augmenter leurs chances d'obtenir un emploi. Les données de l'enquête vont dans le même sens. Ainsi une conseillère a dit explicitement à Isabelle (France) de ne mettre " nulle part sur son CV " qu'elle est médecin, si elle voulait trouver un emploi. Et nombreux sont ceux à avoir usé de cette stratégie. Par exemple, Sayed (Algérie), qui a travaillé dans une manufacture au début de sa trajectoire, dit avoir " appris à ne pas dire [qu'il était] médecin parce qu'on ne [le] prenait jamais " ou Yanet (Cuba), préposée aux bénéficiaires durant les premières années au Québec : "Mais je n'ai pas dit que j'étais médecin, parce que j'avais peur qu'à cause de mon statut de médecin ils me bloquent".

Qu'est-ce qui distingue les DIM réorientés qui révéleront qu'ils sont médecins à leurs employeurs ou à leurs collègues de ceux qui l'omettront ? Dans notre enquête, le niveau d'emploi et le statut professionnel du poste font la différence. En effet, dans les cas de "forte déqualification " (Chicha 2009), c'est-à-dire pour I'obtention d'un emploi exigeant peu d'études, les stratégies d'omission de son statut de médecin sont courantes, par exemple pour ceux qui ont travaillé comme manœuvre, préposé aux bénéficiaires, et parfois dans une profession technique en santé. De façon similaire, la recherche de Vapor et $\mathrm{Xu}$ (2011) indique que des médecins réorientés, employés comme infirmier(ères) préfèrent " rester incognito " vis-à-vis des collègues pour éviter de subir des préjudices ou même des formes de discrimination. Le changement de rôle et de statut pourrait être difficile à comprendre pour l'entourage professionnel et entraîner des relations de travail délicates. Ainsi, durant ses études de technique en santé, Sofia ne confiera qu'elle est médecin qu'à quelques collègues de classe proches, mais pas à ses professeurs : " Je ne le dis pas parce que je pense que ça peut... avoir un effet au contraire négatif ". 
À l'inverse, dans le cas de professions pour lesquelles leurs acquis professionnels constituent des avantages qui favoriseront leur embauche, le fait d'être médecin ne leur portera pas préjudice, au contraire. C'est le cas de ceux qui travaillent ou ont travaillé dans la recherche, ou de ceux qui ont amorcé une carrière suite à des études supérieures au cours desquelles leur bagage de médecin pouvait être valorisé. À cet égard, Eduardo atteste même que " ça a aidé au début " que ses collègues sachent qu'il est médecin.

En bref, la présentation de soi sera faite de façon stratégique, selon les avantages ou les risques liés au fait de se dire médecin, selon le contexte, et plus particulièrement, selon le niveau du poste convoité. Le médecin ne tait donc pas son statut parce qu'il a " honte " d'exercer un emploi déqualifié et qu'il souhaite préserver une image positive de soi, mais plutôt pour se protéger vis-à-vis des collègues ou des employeurs, aux yeux desquels être médecin pourrait sembler étrange ou incompréhensible.

\section{« Ne pas faire de vagues »}

$$
\begin{array}{r}
\text { "Il y a ce regard, de... "Ah ! [Vous les DIM] vous êtes faibles ! Vous êtes } \\
\text { mauvais !" "(Catiana, Chili, en résidence). }
\end{array}
$$

Les DIM insérés dans la filière médicale mobilisent une autre stratégie pour gérer leurs relations avec leurs collègues et leurs supérieurs, en particulier au cours de la période de résidence : " ne pas faire de vagues ", être " profil bas " afin de ne pas être étiqueté comme un élément perturbateur et risquer l'exclusion du groupe ou du programme. C'est une réalité pour tout résident qu'il soit diplômé du Québec ou d'ailleurs, toutefois celle-ci semble très marquée pour les résidents $\mathrm{DIM}^{23}$. Plus de la moitié des répondants présents dans la filière médicale ont mentionné spontanément ce type de stratégie ${ }^{24}$. En effet, l'accès à la résidence survient suite à un parcours sélectif et exigeant en termes de temps et énergie. Si près du but, on serait tenté de croire qu'ils sont pratiquement reconnus comme médecin, que la réussite de la résidence est quasi assurée. Cependant, jusqu'à la fin de la résidence, ils vivent dans l'incertitude : I'exclusion du programme est toujours possible et le taux d'échec et de prolongation des études pour les DIM est plus élevé que pour les diplômés nord-américains en médecine (CMQ, 2009). Différentes stratégies en découlent.

Ces médecins diplômés à l'étranger pourront tenter de déjouer ces a priori négatifs attribués aux DIM lorsqu'il est possible de se présenter différemment. Catiana (Chili) qui a changé d'orientation en cours de résidence se sent avantagée du fait que ses superviseurs la considèrent comme " préalablement intégrée au système ". Elle se dit chanceuse - tout en considérant la situation injuste pour les autres DIM - que son patron ne l'associe aux DIM. De son côté,

23 Une étude met en lumière que les résidents DIM reçoivent plus de sanctions disciplinaires que ceux diplômés au Canada (Belluz, 2012).

24 Phénomène aussi constaté pour les aspirants à la résidence. Tous les DIM qui témoignent dans le reportage Médecins sans résidence, de Bellange (2010) " ont refusé de parler à la caméra, par peur de représailles de l'establishment médical " (www.onf.ca/ film/medecins_sans_residence). 
Ana qui a débuté par l'externat, se présente aux autres durant sa résidence comme diplômée de "telle " Université au Québec et non comme DIM pour ne pas être évaluée négativement :

"Même si je suis encore étrangère - parce que ça ne se change pas -, mais moi j'affirme toujours que j'ai fini à l'Université [au Québec]. Parce qu'il y a quand même une discrimination [de la part des superviseurs] qui se fait d'emblée entre les médecins étrangers qui commencent la résidence directement et un médecin qui finit ici les études.

[...] Ils sont d'emblée visés. Mais pas dans le sens d'être aidés, dans le sens de trouver leurs défauts, malheureusement " (Ana, Roumanie, en résidence).

Bien que l'expérience de la résidence ne soit pas vécue négativement par tous les DIM, elle implique un changement de statut et un ajustement de l'identité professionnelle. "Il faut se remettre dans la peau de l'étudiant " dit Sayed, et se couler dans le moule où les relations de pouvoir changent, parfois avec des professeurs plus jeunes que soi. D'autres résidents disent explicitement qu'ils ont appris à ne pas faire de vagues, "à ne rien dire". Ana rapporte par exemple qu'en cas de désaccord avec un patron lors de sa résidence, elle se retient d'exprimer son opinion, même si son expérience antérieure comme médecin l'inciterait à débattre. "Il faut un contrôle mental pour ne pas exprimer à travers notre visage ce qu'on pense ". Pour elle, si l'on veut réussir, il faut " résister " et avoir " beaucoup d'humilité ", et " surtout ne pas entrer dans des divergences d'opinions".

Pour les nouveaux médecins en poste ou pour ceux qui sont passés par un stage d'évaluation pour l'obtention du permis restrictif, on constate une stratégie similaire, mais dans une version positive : le DIM met en avant I'humilité et l'importance d'acquérir de nouvelles connaissances, de s'adapter à un nouveau système. Les interviewés disent ainsi que "j'ai fait l'éponge " (Jean-Claude), "que je m'adapte, que j'observe et que je me taise " (Monique). À l'instar d'Ana, ils attendront de se sentir appuyés et d'obtenir le soutien d'un réseau de collègues avant d'exprimer leurs idées, surtout si elles sont différentes.

Kamila Saieed (2011), qui a témoigné de sa trajectoire d'intégration professionnelle en Alberta comme DIM, fait allusion au "syndrome de la langue liée " des DIM (tongue-tied syndrome), où la perte de confiance en soi durant sa résidence I'amenait à se retirer des interactions. Selon Crutcher et ses collaborateurs (2011), des différences culturelles pourraient expliquer le peu d'interactions des résidents DIM avec leurs professeurs. Des incompréhensions découleraient de cet écart entre le système de santé canadien - qui s'attend à ce que les résidents développent une pensée critique quant au processus de décision clinique - et plusieurs DIM, pour qui la déférence au professeur et la nécessité d'acquiescer avec modestie feraient partie de leur culture. Nous estimons que cette réalité peut être analysée différemment. Les modalités d'interactions peuvent certes être influencées par certaines caractéristiques personnelles du DIM, mais elles peuvent aussi représenter une réaction à un contexte. Dans notre enquête, de nombreux témoignages recueillis évoquent cette "loi du silence " afin d'éviter d'être perçu comme un élément perturbateur ou de se protéger par crainte d'être mal évalué ou interprété. S'agit-il là d'une réaction stratégique dans le but d'éviter une stigmatisation potentielle (Goffman, 1975) ? 


\section{Conclusion}

Les choix et les stratégies professionnelles ne sont pas uniquement attribuables à des qualités intrinsèques, à des valeurs ou à la culture. Le contexte structurel encadrant les possibilités de reconnaissance, les relations de pouvoir ainsi que les ressources sociales et symboliques des migrants modulent les possibilités de s'intégrer dans la filière médicale (Fortin et Renaud, 2004 ; Berset et al., 1999). Les trajectoires des DIM enquêtés illustrent combien I'identité constitue un processus interactif et dynamique qui laisse place à la négociation entre les acteurs en présence. Elles montrent également que cette capacité stratégique n'est pas également distribuée (Sainsaulieu, 1977). Outre les médecins déjà engagés dans des trajectoires prestigieuses et compétitives avant la migration, qui sont toujours en poste lorsqu'ils sont recrutés, les médecins ont presque tous rencontré des obstacles et ont été exposés à des discours leur suggérant de réévaluer leurs attentes, une plus grande "flexibilité ", pouvant aller jusqu'au " deuil " de la médecine. En somme, la capacité stratégique des DIM est contrastée dans un contexte où la mobilisation de ressources sociales et symboliques est centrale.

L'identité pour soi n'explique que de façon très parcellaire les trajectoires d'intégration professionnelle. Les conditions d'appartenance et l'attribution identitaire influencent les trajectoires et entraînent des stratégies de retrait de la profession ou du pays, de négociation identitaire et de relativisation. Du point de vue identitaire, les DIM réorientés dans des techniques en santé peuvent taire leur ancien statut de médecin afin de se protéger des réactions négatives de collègues ou d'employeurs, tandis que ceux qui cherchent à s'intégrer dans " I'establishment médical " se distancient parfois de I'identité de groupe DIM qui pourrait les stigmatiser. Paradoxalement, tandis que les DIM aspirant à la résidence doivent compter sur le soutien de leurs pairs afin d'élaborer des stratégies complexes pour s'intégrer au système médical québécois, cette identité de groupe pourrait les défavoriser, particulièrement en situation de militance pour la reconnaissance.

La lutte pour la reconnaissance professionnelle prend forme dans un contexte hautement compétitif, dont les règles requièrent une vision de l'intérieur. Affiché comme un processus neutre, il apparaît au contraire que le caractère idéologique, relationnel et subjectif le sous-tend. Dans quelle mesure le " capital humain " peut-il expliquer des trajectoires si hétérogènes au sein d'un même groupe professionnel ? La reproduction sociale et organisationnelle écarte les sujets qui ne reflètent pas "la norme "(Sainsaulieu, 1997). Face à cela, les médecins déploient un ensemble de stratégies pour se protéger, pour retrouver un certain équilibre en regard de leurs nouveaux choix professionnels ou pour éviter d'être stigmatisés. L'identité pour soi apparaît dès lors comme un élément relativement réducteur pour expliquer des trajectoires d'intégration, tout comme les caractéristiques personnelles des migrants. Être diplômé international en médecine et s'intégrer dans le système médical est possible au Québec, mais le plus souvent leur identité professionnelle est malmenée et leur trajectoire parsemée d'épreuves et d'incertitudes. 


\section{Références bibliographiques}

Bardaï Anas (2010) L'intégration professionnelle : perception des médecins immigrants, Maîtrise en administration des services de santé, Université de Montréal, $114 \mathrm{p}$.

Becker Howard S., Geer Blanche, Hughes Everett C. and Strauss Anselm L. (2007 [1961]) Boys in White: Student Culture in Medical School, 10e éd., New Brunswick \& London, Transaction Publishers, 456 p.

Belkhodja Chedly, Forgues Éric, Gaboury Isabelle, Guignard Noël Josée, Bahi Boniface, Nkolo Christine et Tawil Nada (2009) L'intégration des diplômés internationaux en santé francophones dans les communautés francophones en situation minoritaire. Rapport final de recherche, Consortium national de formation en santé \& Institut canadien de recherche sur les minorités linguistiques, 63 p., [en ligne] consulté le 14/05/2012. URL : http://cnfs.net/fr/publications-immigration.php?publication $=716$

Belluz Julia (2012) Why do IMGs see more discipline cases?, The Medical Post, 48 (6), pp. 8-13.

Berset Alain, Weygold Serge-Alexandre, Crevoisier Olivier et Hainard François (1999) Qualification-déqualification professionnelle des immigrants. Approche théorique, Revue Européenne des Migrations Internationales, 15 (3), pp. 87-100.

Blain Marie-Jeanne (2006) Trajectoires socioprofessionnelles et processus identitaires en contexte de migration : de la Colombie au Québec, Maîtrise en anthropologie, Université de Montréal, $215 \mathrm{p}$.

Blain Marie-Jeanne, Suárez-Herrera José Carlos et Fortin Sylvie (2012) L'intégration professionnelle de médecins diplômés à l'étranger au Québec : un enjeu d'envergure en santé mondiale, Anthropologie et santé, 5, 16 p., [en ligne] consulté le 03/11/2012. URL : http://anthropologiesante.revues.org/973

Boyd Monica et Schellenberg Grant (2007) Réagrément et professions des médecins et ingénieurs immigrants, Tendances sociales canadiennes, 11 (008), $11 \mathrm{p}$.

Canadian Resident Matching Service (CaRMS) (2013) 2013 R-1 Match. Table 50: IMGs by Region of Graduation, [en ligne] consulté le 15/11/2013. URL : www. carms.ca

Chicha Marie-Thérèse (2009) Le mirage de l'égalité : les immigrées hautement qualifiées à Montréal, La fondation canadienne des relations raciales, 136 p.

Chicha Marie-Thérèse et Charest Éric (2008) L'intégration des immigrés sur le marché du travail à Montréal : politiques et enjeux, Choix IRPP, 14 (2), 62 p.

Citoyenneté et Immigration Canada (CIC) (2011) La catégorie de I'expérience canadienne attire les meilleurs et les plus brillants au Canada, Communiqué de presse, [en ligne] consulté le 20/11/2011. URL : http://nouvelles.gc.ca/web/articlefr.do?nid=635009

Collège des Médecins du Québec (CMO) (2013) Statistiques. Les médecins du Québec - Répartition au 31 mai 2013, [en ligne] consulté le 30/10/2013. URL : www.cmq.org 
Collège des Médecins du Québec (CMQ) (2009) Prolongations : comparaison entre les résidents DHCEU et les résidents non-DHCEU du contingent régulier des cohortes 1994 à 2007, dans des programmes de formation postdoctorale en médecine familiale ou spécialisée, [en ligne] consulté le 24/05/2011. URL : www. cmq.org

Collins John (2012) New Normalising: A Grounded Theory of the Transition for Migrant Health Care Professionals, Doctor of Philosophy, School of Education Studies, Dublin City University, 222 p.

Commission des Droits de la Personne et des Droits de la Jeunesse du Québec (CDPDJ) (2010) Dossier : MTL-018303. Enquête de la propre initiative de la Commission des droits de la personne et des droits de la jeunesse, en vertu de l'article $711^{\circ}$ de la Charte des droits et libertés de la personne, 16 p., [en ligne] consulté le 02/04/2012. URL : http://www.cdpdj.qc.ca/Documents/ Communication_resolution_COM_559_5_1_1_Arial.pdf

Conseil Interprofessionnel du Québec (CIQ) (2012) Les professions réglementées, [en ligne] consulté le 12/08/2012. URL : http://www.professions-quebec.org/ index.php/fr/element/visualiser/id/62

Crutcher Rodney, Mann Peggy, Baumber John, Hofmeister Marianna and Armson Heather (2011) The merging of Cultures. The Alberta International Medical Graduate Program Experience, in Earle H. Waugh, Olga Szafran and Rodney Crutcher Eds., At the Interface of Culture and Medicine, Edmonton, University of Alberta Press, pp. 75-106.

Dubar Claude (1995) La socialisation. Construction des identités sociales et professionnelles, 2e éd., Paris, Armand Colin, 276 p.

Dumont Jean-Christophe et Zurn Pascal (2007) Les personnels de santé immigrés dans les pays de I'OCDE dans le contexte général des migrations de travailleurs hautement qualifiés, Perspectives des migrations internationales 2007, SOPEMI, OCDE, pp. 171-244, [en ligne] consulté le 20/11/2008. URL : http:// www.oecd.org/fr/migrations/mig/41515734.pdf

Dussault Gilles (1985) Les effectifs sanitaires au Québec, in Jacques Dufresne, Fernand Dumont etYves Martin Éds., Traité d'anthropologie médicale. L'institution de la santé et de la maladie, Québec, PUQ, IQRC, PUL, pp. 587-603.

Fortin Sylvie et Renaud Jean (2004) Stratégies d'établissement en contexte montréalais : une diversité de modalités ?, in Jean Renaud, Annick Germain et Xavier Leloup Dir., Racisme et discrimination : les tensions et les défis d'une société plurielle, Québec, Les Presses de I'Université Laval, pp. 31-59.

Fournier Geneviève et Bourassa Bruno (Dir.) (2004) La précarité professionnelle : effets individuels et sociaux. Entretiens du CRIEVAT-Laval avec Serge Paugam, Saint-Nicolas, Les Presses de I'Université Laval, 101 p.

Galarneau Diane et Morissette René (2004) Les immigrants sont-ils perdants ?, Perspective, 75-001-XIF, $17 \mathrm{p}$.

Gallissot René (1987) Sous l'identité, le procès d'identification, L'Homme et la Société, 83, pp. 12-27.

Goffman Erving (1975) Stigmate. Les usages sociaux des handicaps, Paris, Les Éditions de Minuit, 175 p. 
Good J. Byron and Delvecchio Good Mary-Jo (1993) Learning Medicine. The construction of Medical Knowledge at Harvard Medical School, in Shirley Lindenbaum and Margaret Lock Eds., Knowledge, Power and Practice. The anthropology of Everyday Life, Berkeley, University of California Press, pp. 81-107.

Hachimi Alaoui Myriam (2007) Les chemins de l'exil : les Algériens exilés en France et au Canada depuis les années 90, Paris, L'Harmattan, 200 p.

Institut Canadien d'Information sur la Santé (ICIS) (2013) Nombre, répartition et migration des médecins canadiens 2012, 158 p., [en ligne] consulté le 30/10/2013. URL : www.cihi.ca

Lamontagne Yves (2005) Éditorial, Le collège, XLV (1), p. 5.

Ministère de I'Immigration et des Communautés Culturelles (MICC) (2012) Obstacles rencontrés par les immigrants pour trouver un emploi, [en ligne] consulté le 15/05/2012. URL : http://www.immigration-quebec.gouv.qc.ca/fr/ immigrer-installer/travailleurs-permanents/demande-immigration-general/ obstacles-emploi.html

Neiterman Elena and Bourgeault Ivy Lynn (2012) Conceptualizing Professional Diaspora: International Medical Graduates in Canada, Migration \& Integration, 13, pp. 39-57.

Organisation Mondiale de la Santé (OMS) (2010) Code de pratique mondial de I'OMS pour le recrutement international des personnels de santé, 73e Assemblée mondiale de la Santé, WHA63.16, 14 p.

Remennick Larissa and Shakhar Gila (2003) You Never Stop Being a Doctor: The Stories of Russian Immigrant Physicians Who Converted to Physiotherapy, Health, 7 (1), pp. 87-108.

Saieed Kamila (2011) Kamila's Reflection, in Earle H. Waugh, Olga Szafran and Rodney A. Crutcher Eds., At the Interface of Culture and Medicine, Edmonton, University of Alberta Press, pp. 69-73.

Sainsaulieu Renaud (1977) Identités collectives et reconnaissance de soi dans le travail, in Renaud Sainsaulieu, L'identité au travail : les effets culturels de I'organisation, Paris, Presses de la Fondation nationale des sciences politiques, pp. 302-343.

Sainsaulieu Renaud (1997) Sociologie de l'entreprise : organisation, culture et développement, $2 \mathrm{e}$ éd., Paris, Presses de Sciences Po, 476 p.

Séchet Raymonde et Vasilcu Despina (2012) Les migrations de médecins roumains vers la France, entre démographie médicale et quête de meilleures conditions d'exercice, Norois, 2 (223), pp. 63-76.

Shuval Judith T. (2000) The Reconstruction of Professional Identity among Immigrant Physicians in Three Societies, Journal of Immigrant Health, 2 (4), pp. 191-202.

Shuval Judith T. and Bernstein Judith H. (Dir.) (1997) Immigrant Physicians: Former Soviet Doctors in Israel, Canada, and the United States, Wesport, Connecticut, London, Praeger Publishers, 200 p.

Shuval Judith T. and Bernstein Judith H. (1996) The Dynamics of Professional Commitment: Immigrant Physicians from the Former Soviet Union in Israel, Social Science \& Medicine, 42 (7), pp. 965-974. 
Smith Micheal P. and Favell Adrian (Eds.) (2006) The Human Face of Global Mobility, New Brunswick, Transaction Publishers, 314 p.

Sow Doudou (2010) Un autre discours sur l'intégration des médecins diplômés à I'étranger est possible, [en ligne] consulté le 01/09/2012. URL : http://doudousow. wordpress.com/2010/11/17/un-autre-discours-sur-I\%E2\%80\%99integration-desmedecins-diplomes-a-I\%E2\%80\%99etranger-est-possible/

Türegün Adnan (2011) What Do Immigrants Do When They Can't Practise Their Professions? Immigrant Professionals in the Ontario Settlement Service Sector, Toronto, CERIS-The Ontario Metropolis Centre, $43 \mathrm{p}$.

Vapor Victor R. and Xu Yu (2011) Double Whammy for a New Breed of ForeignEducated Nurses: Lived Experiences of Filipino Physician-Turned Nurses in the United States, Research and Theory for Nursing Practice, 25 (3), pp. 210-226. 


\section{Marie-Jeanne Blain, Sylvie Fortin et Fernando Alvarez}

\section{$\cdots$ Être médecin et immigrant au Québec : une identité professionnelle malmenée}

Au Québec et au Canada, les politiques d'immigration visent à attirer "les meilleurs et les plus brillants ". Les médecins font partie de cette élite transnationale et leur "fuite " soulève des enjeux éthiques dans les zones mal desservies. Or, un second enjeu éthique apparaît au pays hôte : leur reconnaissance professionnelle parcellaire. Elle soulève là encore de nombreux défis pour ces médecins, dont celui d'une identité professionnelle malmenée, qui reste pourtant peu documentée. Le caractère dynamique et relationnel de l'identité professionnelle est mis en relief, mais surtout, la puissance des conditions d'appartenance qui oblige à une flexibilité professionnelle et parfois au retrait de la profession ou du pays ; la notion de pouvoir et celle de reconnaissance sont au cœur de ces processus identitaires. Dans cet article les auteurs posent un regard critique sur les approches centrées sur l'individu qui ignorent les normes et les contraintes sociales.

\section{Being a Physician and an Immigrant in Quebec: A Professional Identity Mistreated}

In Quebec and Canada, immigration policies are designed to attract "the best and the brightest." Physicians are part of this transnational elite and the "brain drain" raises ethical issues in under-served areas. A second ethical issue appears in the host country: Their fragmented professional recognition. In this interplay for recognition, the identity of doctors is often mistreated. Our starting point is the testimony of migrants; the dynamic and relational nature of professional identity is emphasized, but more importantly, the force of the conditions membership that require professional flexibility and, at times, the withdrawal of the profession or country. The notion of power and recognition is at the heart of identity processes. We take a critical approach to the individual-centered approaches that ignore social norms and constraints.

\section{Ser médico e inmigrante en Quebec: una identidad profesional menospreciada}

Tanto en Quebec como en Canadá, las políticas de inmigración aspiran a atraer «los mejores y los más brillantes». Los médicos forman parte de esta élite transnacional y su "fuga" pone en juego problemas éticos en las zonas mal atendidas. Un segundo problema ético aparece en el país de acogida: el reconocimiento profesional parcelario. Nuestro punto de partida es el testimonio de los migrantes. En este esfuerzo para obtener el reconocimiento, la identidad de los médicos se ve, a menudo, menospreciada. El carácter dinámico y relacional de la identidad profesional se discute, pero sobretodo, la fuerza de las condiciones de pertenencia que imponen una flexibilidad profesional y a veces el abandono de la profesión o del país. El concepto de poder y de reconocimiento está en el centro de los procesos de identidad. Partimos de una mirada crítica hacia las perspectivas enfocadas sobre el individuo, las cuales hacen abstracción de las normas y de los impedimentos sociales. 